\title{
Coupling effect analysis of a conductive bar-shell in a magnetic field
}

\author{
Yuhong Bian" ${ }^{\mathrm{a}}$, Qun You ${ }^{\mathrm{b}}$ and Chen Zhang ${ }^{\mathrm{c}}$
}

Key Laboratory of Mechanical Reliability for Heavy Equipments and Large Structures of Hebei Province, College of Civil Engineering and Mechanics, Yanshan University, Qinhuangdao, China

abianyuhong@163.com, b15032331382@163.com, c18233569194@163.com

Keywords: conductive bar-shell, magnetoelasticity, coupling effect, Lorentz force, discrete-orthogonalization method

Abstract: The nonlinear magnetoelastic problems for a bar-shell in a magnetic field subjected to mechanical loadings are studied. Based on the electrodynamics equations, magnetoelastic kinetic equations, geometric equations, and physical equations of a bar-shell, normal Cauchy form nonlinear differential equations, which include ten basic functions in all, are obtained by the variable replacement method. Using the difference and quasi-linearization methods, the nonlinear magnetoelastic equations are reduced to a sequence of quasilinear differential equations, which can be solved by the discrete-orthogonalization method. Numerical solutions for stresses and deformations in a bar-shell under the interaction of an electromagnetic field and a mechanical load are obtained by considering a specific example. The magnetoelastic effect on the shell by the side electric current and the magnetic induction intensity is studied. The results show that the stress-strain state in the shells can be controlled by changing the electromagnetic parameters.

\section{Introduction}

The magnetoelastic theory studies the mechanical behaviors of electromagnetic structures in electromagnetic fields, the mechanical properties such as the deformation of structures, intensity, vibration, and stability, etc.. It comes down to the subjects such as electromagnetic physics, solid mechanics, etc.. Applications of electromagnetic structures are increasingly broad in modern technological fields, such as aerospace industry, magnetic suspension transportation, high-speed transmission, electromechanical power equipment, etc.. In recent years, the electromagnetic elastic mechanics develops very quickly. Many important achievements are obtained [1-8]. These research achievements laid a good foundation for studies on the electromagnetic elastic mechanics and its applications. However, so far the studies of the nonlinear theory are not enough complete. Except for the problems about the vibration and stability of the structures such as rods, beams, plates, shells, etc. in electromagnetic fields, the analysis of the stress-strain state in the problems is rare. Therefore, the researches on the nonlinear magnetoelastic problems of conductive plates and shells have recently become one of the most important topics for mechanics operators.

In this paper, the nonlinear magnetoelastic problems for a conductive bar-shell under the interaction of an electromagnetic field and a mechanical field are studied. Using the difference and quasi-linearization methods, we have reduced nonlinear partial differential equations with ten basic unknown functions to a sequence of quasi-linear differential equations, which can be solved by the discrete-orthogonalization method. Numerical solutions for magnetoelastic stresses and deformations in a conductive bar-shell are obtained by considering a specific example. The magnetoelastic effect on the shell by the side electric current and the magnetic induction intensity is studied.

\section{Fundamental Equations}

We consider a bar-shell moving in the applied magnetic field. The shell, the mechanical loads, and the electric current distribution in an orthogonal curvilinear coordinate system $\alpha, \beta, \gamma$ are shown in Fig. 1. By satisfying the magnetoelastic supposition of the thin shell [5] and using the theory of elastic mechanics, electromagnetic basic theories [9], we obtain the electrodynamics 
equations, magnetoelastic kinetic equations, geometric equations, and physical equations for a bar-shell.

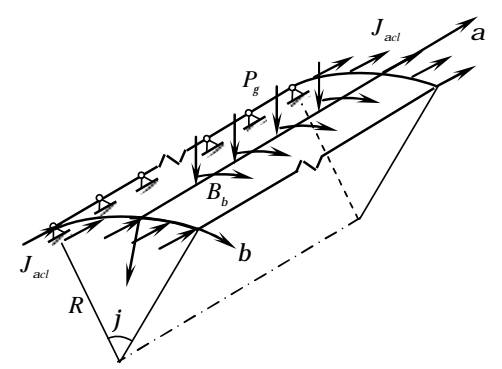

Fig. 1 Distribution diagram of the electric current density and magnetic induction intensity on a bar-shell

The electrodynamics equations are given by

$$
\begin{aligned}
& -\frac{\partial B_{\gamma}}{\partial t}=\frac{\partial E_{\beta}}{\partial \alpha}-\frac{1}{R} \frac{\partial E_{\alpha}}{\partial \beta} \\
& \sigma\left[E_{\alpha}+\frac{\partial v}{\partial t} B_{\gamma}-\frac{1}{2} \frac{\partial w}{\partial t}\left(B_{\beta}^{+}+B_{\beta}^{-}\right)\right]=\frac{1}{R} \frac{\partial H_{\gamma}}{\partial \beta}-\frac{H_{\beta}^{+}-H_{\beta}^{-}}{h}, \\
& \sigma\left[E_{\beta}-\frac{\partial u}{\partial t} B_{\gamma}+\frac{1}{2} \frac{\partial w}{\partial t}\left(B_{\alpha}^{+}+B_{\alpha}^{-}\right)\right]=-\frac{\partial H_{\gamma}}{\partial \alpha}+\frac{H_{\alpha}^{+}-H_{\alpha}^{-}}{R h}
\end{aligned}
$$

The magnetoelastic kinetic equations are given by

$$
\begin{aligned}
& \frac{\partial N_{\alpha}}{\partial \alpha}+\frac{\partial S}{R \partial \beta}+P_{\alpha}+f_{\alpha}=\rho h \frac{\partial^{2} u}{\partial t^{2}}, \\
& \frac{\partial N_{\beta}}{R \partial \beta}+\frac{\partial S}{\partial \alpha}+\frac{\partial M_{\alpha \beta}}{R \partial \alpha}+\frac{Q_{\beta}}{R}+P_{\beta}+f_{\beta}=\rho h \frac{\partial^{2} v}{\partial t^{2}}, \\
& \frac{\partial\left(Q_{\alpha}-N_{\alpha} \theta_{\alpha}-S \theta_{\beta}\right)}{\partial \alpha}+\frac{\partial\left(Q_{\beta}-N_{\beta} \theta_{\beta}-S \theta_{\alpha}\right)}{R \partial \beta}-\frac{1}{R} N_{\beta}+P_{\gamma}+f_{\gamma}=\rho h \frac{\partial^{2} w}{\partial t^{2}}, \\
& \frac{\partial M_{\alpha}}{\partial \alpha}+\frac{\partial M_{\alpha \beta}}{R \partial \beta}-Q_{\alpha}=\frac{\rho h^{3}}{12} \frac{\partial^{2} \theta_{\alpha}}{\partial t^{2}}, \\
& \frac{\partial M_{\beta}}{R \partial \beta}+\frac{\partial M_{\alpha \beta}}{\partial \alpha}-Q_{\beta}=\frac{\rho h^{3}}{12} \frac{\partial^{2} \theta_{\beta}}{\partial t^{2}},
\end{aligned}
$$

where $E_{\alpha}$ and $E_{\beta}$ are the electric field intensities along the corresponding directions; $H_{\gamma}$ and $B_{\gamma}$ are the magnetic field intensity and magnetic induction intensity along the $\gamma$-direction, respectively; $h$ is the thickness of the shell; $R$ is the radius of the shell; $t$ is the time variable; $\sigma$ is the electrical conductivity of the material; $\rho$ is the mass density of the medium; $B_{i}^{ \pm}$and $H_{i}^{ \pm} \quad(i=\alpha, \beta)$ are the values of $B_{i}$ and $H_{i}$ on the upper and lower surfaces of the shell, respectively; $N_{\alpha}, N_{\beta}, Q_{\alpha}, Q_{\beta}, S, M_{\alpha}, M_{\beta}$, and $M_{\alpha \beta}$ are the internal forces and moments along the corresponding directions in the shell; $P_{\alpha}, P_{\beta}$, and $P_{\gamma}$ are the mechanical loads; $f_{\alpha}$, $f_{\beta}$, and $f_{\gamma}$ are the Lorentz forces along the corresponding directions; $u, v$, and $w$ are the displacements along the $\alpha, \beta$, and $\gamma$-directions, respectively; $\theta_{\alpha}$ and $\theta_{\beta}$ are the angles of rotation. 


\section{Computational Method}

Selecting $u, v, w, \theta_{\beta}, N_{\beta}, \hat{Q}_{\beta}, S, M_{\beta}, E_{\alpha}$, and $B_{\gamma}$ as basic unknown functions, uniting the equations (1)-(8), at the same time, considering the geometric equations and physical equations, the magnetoelastic coupling equations, which can be written as follows boundary-value problems, are obtained.

$$
\frac{\partial \boldsymbol{Y}}{\partial \beta}=\boldsymbol{F}(\alpha, \beta, \boldsymbol{Y}) \quad\left(\beta_{1} \leq \beta \leq \beta_{2}\right), \quad C_{1} \boldsymbol{Y}\left(\beta_{1}\right)=\boldsymbol{c}_{1}, C_{2} \boldsymbol{Y}\left(\beta_{2}\right)=\boldsymbol{c}_{2},
$$

where $\boldsymbol{Y}=\left\{u, v, w, \theta_{\beta}, N_{\beta}, \hat{Q}_{\beta}, S, M_{\beta}, E_{\alpha}, B_{\gamma}\right\}^{\mathrm{T}} ; C_{1}$ and $C_{2}$ are given orthogonal matrixes; $\boldsymbol{c}_{1}$ and $\boldsymbol{c}_{2}$ are given vectors.

Problems (9) are reduced to a difference form along the $\alpha$-direction, at the $i$ node, by writing the partial derivatives $\frac{\partial \boldsymbol{Y}}{\partial \alpha}, \frac{\partial^{2} \boldsymbol{Y}}{\partial \alpha^{2}}, \frac{\partial^{3} \boldsymbol{Y}}{\partial \alpha^{3}}, \frac{\partial^{4} \boldsymbol{Y}}{\partial \alpha^{4}}$ with respect to $\alpha$ as [10], at the same time, Newmark's stable finite equidifferent formulas [5] are used to find the derivatives with respect to time in the magnetoelastic coupling equations for a time step length, thus, equations (9) can be expressed as

$$
\frac{\mathrm{d} \boldsymbol{Y}}{\mathrm{d} \beta}=\boldsymbol{F}(\beta, \boldsymbol{Y}) \quad\left(\beta_{1} \leq \beta \leq \beta_{2}\right), \quad C_{1} \boldsymbol{Y}\left(\beta_{1}\right)=\boldsymbol{c}_{1}, \quad C_{2} \boldsymbol{Y}\left(\beta_{2}\right)=\boldsymbol{c}_{2} .
$$

The problems described by equations (10) are nonlinear. With the linearization method, nonlinear problems can be turned into a series of linear problems, thus, we obtain a set of linear ordinary differential equations. For the corresponding initial and boundary conditions, all unknown variables can be found by the discrete-orthogonalization method.

\section{Numerical Examples}

Figure 1 shows a shell made of aluminium in a magnetic field $\boldsymbol{B}=\left\{0, B_{\beta}, 0\right\}$. The density of side current in the shell is $\boldsymbol{J}_{c l}=\left\{J_{\alpha c l}, 0,0\right\}$, the mechanical load is $\boldsymbol{P}=\left\{0,0, P_{\gamma}\right\}$. Let $E=7.1 \times 10^{10} \mathrm{~N} / \mathrm{m}^{2}, v=0.3, \rho=2670 \mathrm{~kg} / \mathrm{m}^{3}, \sigma=3.63 \times 10^{7}(\Omega \cdot \mathrm{m})^{-1}, \mu=1.256 \times 10^{-6} \mathrm{H} / \mathrm{m}$, $J_{\alpha c l}=J_{\alpha} \sin \omega t \mathrm{~A} / \mathrm{m}^{2}, \omega=\pi \times 10^{2} \mathrm{~s}^{-1}, \quad P_{\gamma}=50 \mathrm{~N} / \mathrm{m}^{2}, \quad h=5 \times 10^{-3} \mathrm{~m}, \quad R=1 \mathrm{~m}$, the opening angle of the shell is $\varphi=\pi / 6$.

The initial conditions and the boundary conditions are

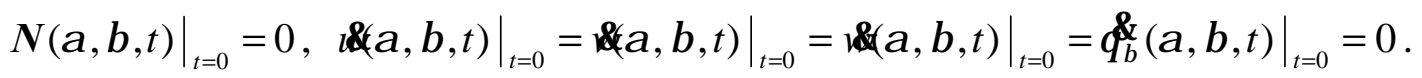

$$
\begin{aligned}
& \beta=-\pi / 12: \quad B_{\gamma}=0.1 \sin \omega t \mathrm{~T}, u=v=w=0, M_{\beta}=0 \text {; } \\
& \beta=\pi / 12: B_{\gamma}=0, N_{\beta}=0, \hat{Q}_{\beta}=0, S=0, M_{\beta}=0 \text {. }
\end{aligned}
$$

Effect on the Deformation of the Bar-shell by the Electromagnetic Parameters. Figure 2 shows the deflection distribution in the $\beta$-direction for $P_{\gamma}=500 \mathrm{~N} / \mathrm{m}^{2}, J_{\alpha}=2 \mathrm{MA} / \mathrm{m}^{2}$, $B_{\beta}=0.2 \mathrm{~T}$, and $t=20 \mathrm{~ms}$. Figure 3 shows the deflection distribution in the $\beta$-direction for $J_{\alpha}=2 \mathrm{MA} / \mathrm{m}^{2}, t=19 \mathrm{~ms}$, and different magnetic induction intensity. Figure 4 shows the deflection distribution in the $\beta$-direction for $B_{\beta}=0.2 \mathrm{~T}, t=19 \mathrm{~ms}$, and different electric current 
density. Figure 5 shows the variation of the deflection $(\beta=0)$ with time for $J_{\alpha}=2 \mathrm{MA} / \mathrm{m}^{2}$ and different magnetic induction intensity. Figure 6 shows the variation of the deflection $(\beta=0)$ with time for $B_{\beta}=0.2 \mathrm{~T}$ and different electric current density.

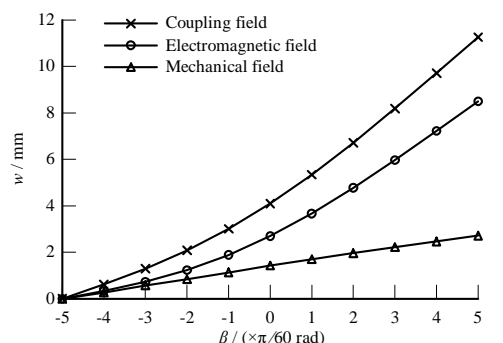

Fig. 2 Contrast diagrams of the deflection.

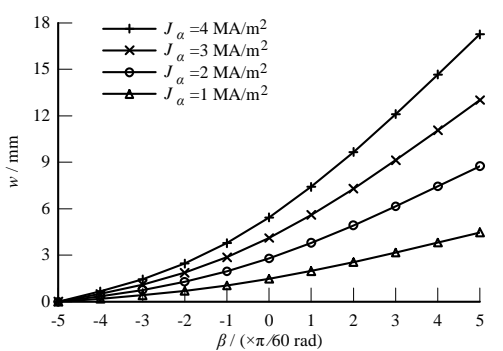

Fig. 4 Curves of $w$ distribution for different $J_{\alpha}$.

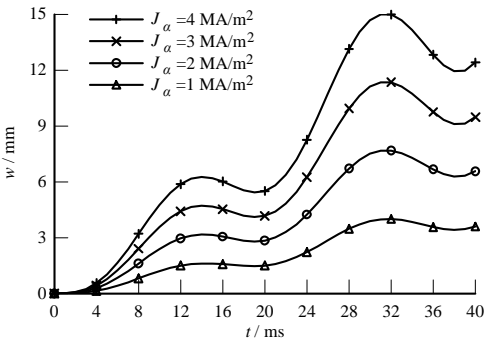

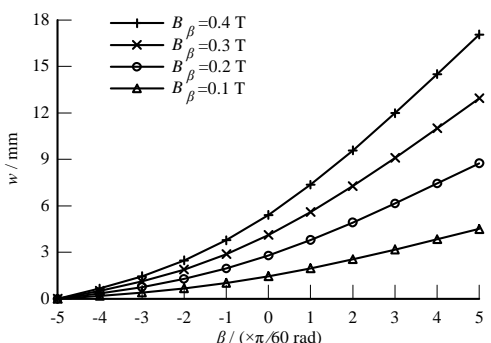

Fig. 3 Curves of $w$ distribution for different $B_{\beta}$.

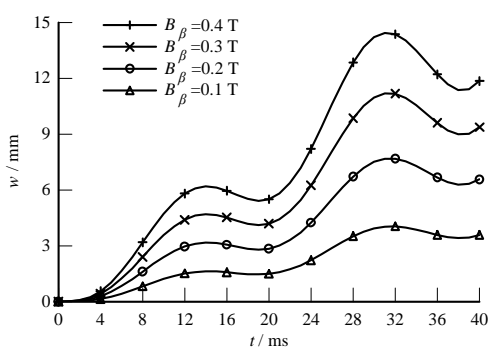

Fig. 5 The $w(\beta=0)$ versus $t$ for different $B_{\beta}$.

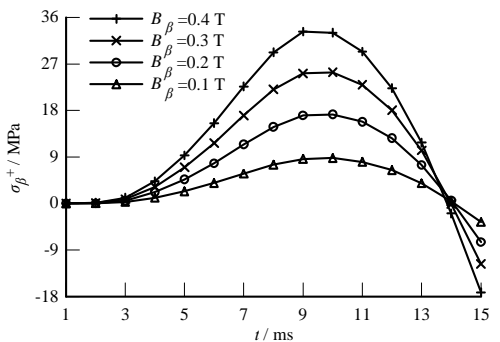

Fig. 6 The $w(\beta=0)$ versus $t$ for different $J_{\alpha}$. Fig. 7 The $\sigma_{\beta}^{+}(\beta=0)$ versus $t$ for different $B_{\beta}$. Effect on the Stress of the Bar-shell by the Electromagnetic Parameters. Figure 7 shows the variation of the normal stress $\sigma_{\beta}^{+}$in the $\beta$-direction on the upper surface $(\beta=0)$ with time for $J_{\alpha}=4 \mathrm{MA} / \mathrm{m}^{2}$ and different magnetic induction intensity. Figure 8 shows the variation of the normal stress $\sigma_{\beta}^{+}$in the $\beta$-direction on the upper surface $(\beta=0)$ with time for $B_{\beta}=0.2 \mathrm{~T}$ and different electric current density. Figure 9 shows the stress distribution for $J_{\alpha}=4 \mathrm{MA} / \mathrm{m}^{2}$, $B_{\beta}=0.4 \mathrm{~T}$, and $t=10 \mathrm{~ms}$. Curves $a$ and $b$ are the stresses $\sigma_{\alpha}^{+}, \sigma_{\alpha}^{-}$in the $\alpha$-direction on the upper, lower surfaces of the shell, respectively; curves $c$ and $d$ are the stresses $\sigma_{\beta}^{+}, \sigma_{\beta}^{-}$in the $\beta$-direction on the upper, lower surfaces of the shell, respectively.

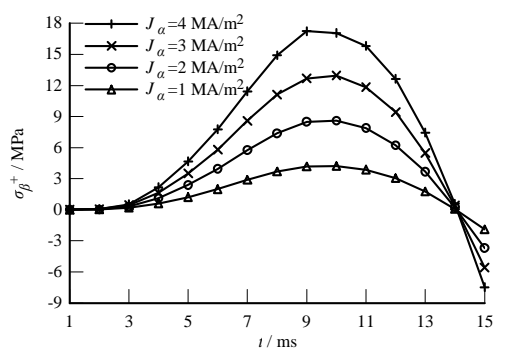

Fig. 8 The $\sigma_{\beta}^{+}(\beta=0)$ versus $t$ for different $J_{\alpha}$.

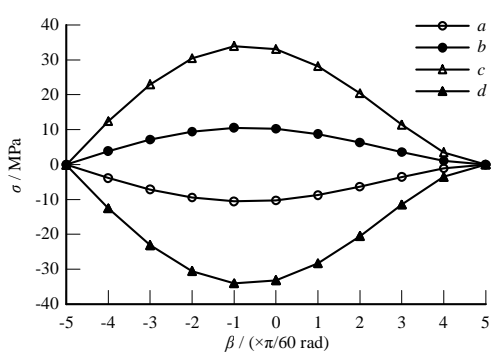

Fig. 9 Curves of the stress distribution. 


\section{Conclusions}

The nonlinear magnetoelastic problems for a bar-shell in a magnetic field subjected to mechanical loadings have been studied in this paper. By the results, we now know that:

(1) the kinetic behavior of the bar-shells can be changed by the magnetic induction intensity or electric current density;

(2) the stresses and deformations in a bar-shell nonlinearly increase with the magnetic induction intensity or electric current density;

(3) the stress-strain state in the shells can be controlled by changing electromagnetic parameters.

\section{Acknowledgements}

This work was financially supported by the National Natural Science Foundation of China (50675186).

\section{References}

[1] X. J. Zheng, J. P. Zhang, and Y. H. Zhou, Dynamic stability of a cantilever conductive plate in transverse impulsive magnetic field, Int. J. Solids Struct., 42 (2005) 2417-2430.

[2] B. Pratiher, Non-linear response of a magneto-elastic translating beam with prismatic joint for higher resonance conditions, International Journal of Non-Linear Mechanics, 46 (2011) 685-692.

[3] D. J. Hasanyan, L. Librescu, and D. R. Ambur, Buckling and postbuckling of magnetoelastic flat plates carrying an electric current, Int. J. Solids Struct., 43 (2006) 4971-4996.

[4] Y. H. Bian, Analysis of nonlinear stresses and strains in a thin current-carrying elastic plate, Int. Appl. Mech., 51 (2015) 108-120.

[5] L. V. Mol'chenko, Nonlinear magnetoelasticity of thin current-carrying shells, Vyshch. Shkola, Kyiv, 1989.

[6] L. V. Mol'chenko and I. I. Loos, The stress state of a flexible orthotropic spherical shell subject to external current and mechanical force in a magnetic field, Int. Appl. Mech., 49 (2013) 528-533.

[7] S. Soni, N. K. Jain, and P. V. Joshi, Analytical modeling for nonlinear vibration analysis of partially cracked thin magneto-electro-elastic plate coupled with fluid, Nonlinear Dynamics, 90 (2017) 137-170.

[8] M. Mohammadimehr and R. Rostami, Bending and vibration analyses of a rotating sandwich cylindrical shell considering nanocomposite core and piezoelectric layers subjected to thermal and magnetic fields, Applied Mathematics and Mechanics, 39 (2018) 219-240.

[9] F. C. Yu and C. K. Zheng, Electrodynamics, Peking University Press, Beijing, 2004.

[10]Z. L. Xu, Elastic mechanics, High Education Press, Beijing, 2006. 